Int J Infect Microbiol 2012;1(2):41-42

\section{Antimicrobial resistance: a global threat}

\section{Thapa B}

Kathmandu Medical College Teaching Hospital, Kathmandu, Nepal, Genesis Laboratory and Research, Kathmandu, Nepal

email: badri_bishal@yahoo.com, Tel. No.: (+97)9841220108
Since the discovery of penicillin by Sr. Alexender Fleming in 1928, it had been instrumental in treating critically ill patients and increasing life expectancy. However the emergence of penicillin resistance in 1940s has threatened all the gains offered by it. Newer pharmaceutical companies emerged and newer antimicrobial agents were discovered and commercialized for the treatment of infections but most of them are already ineffective to treat infections due to growing antimicrobial resistance. This emergence of multidrug (MDR), pan-drug (PDR) and extensively-drug resistant (XDR) pathogens is a global problem, the seriousness of which is evident by the fact that WHO adopted the World Health Day theme 2011 as "Combating antimicrobial resistance". The growing resistance has posed serious effects for the health care systems in addition to the economic burden to the patients and families.

Almost one third of world's population is infected with tuberculosis and it's a public health problem. Emergence of TB among HIV infected has worsened the scenario of both the diseases. Malaria is another disease of the poor complicating the situation. In addition to these infectious diseases of public health importance numerous bacterial, viral, protozoal and fungal agents are infecting patients in the hospitals and the community. Usually the first line antimicrobial agents cure the patients, but the treatment has to be switched to second line when they emerge as drug resistant. This second line drugs (reserve drugs) are more expensive and more toxic and are also becoming ineffective. The methicillin resistant and vancomycin resistant Staphylocccus aureus, MDR and PDR Acinetobacter baumannii, Pseudomonas aeruginosa, Citrobacter spp., Stenotrophomonas malotphila etc., metallobetalactamase producing E. coli and Klebsiella pneumoniae, antiretroviral resistant HIV-1, MDR and XDR strains of $M$. tuberculosis have already emerged in the globe. ${ }^{1,2,3,4}$ Growing antimicrobial resistance has numerous effects like, prolonged hospital stay, treatment failure, spread of resistant pathogens to other patients, secondary complications and economic, social and mental problems to the patients as well as for their families. The achieved success in controlling TB will be ruined by increasing MDR and XDR strain and is a threat to the TB control programmes. Similarly, emergence of Artesunate resistance in malaria parasite will pose challenge in malaria control. ${ }^{5}$ 
The emergence of resistance is due to inappropriate and indiscriminate use of over the counter antimicrobial agents, irrational use, inadequate infection control practices, poor compliance, over prescription of antimicrobial agents for incentives and ineffective pharmacovigillance systems. Moreover, the emergence of resistance is intimately associated with poverty compelling patients to abort treatment against medical advice. Antimicrobial agents are also becoming popular in agriculture, veterinary and fishery. This wide use of antimicrobials has created selective pressure for human and environmental microorganisms to evolve themselves as resistant. The antimicrobial resistant microorganisms and their resistant genes are growing as an environmental pollutant in the globe.

The universal success on containing antimicrobial resistance is possible through combined efforts on advocacy of rational use of antimicrobial agents, development of effective policy to address the rational use of antimicrobials and its implementation, strengthening antimicrobial surveillance network, rational use of antimicrobials in veterinary, fishery and agriculture, standardization of treatment guidelines and most importantly global commitment, adequate resources allocation and research work in this arena to generate evidence to formulate/guide the policy to address antimicrobial resistance.

\section{REFERENCES}

1. Kumarasamy KK, Toleman MA, Walsh TR, et al. Emergence of a new antibiotic resistance mechanism in India, Pakistan, and the UK: a molecular, biological, and epidemiological study. Lancet Infect Dis 2012;10:597602.

2. David L. Paterson. Resistance in Gram-negative bacteria: Enterobacteriaceae. Am J Infect Control 2006;34:S20-28

3. Louis B. Rice. Antimicrobial resistance in Grampositive bacteria. Am J Med 2006;119:S11-S19

4. Pawlowski A, Jansson M, Sköld M, Rottenberg ME, Källenius G (2012) Tuberculosis and HIV Co-Infection. PLoS Pathog 8(2): e1002464. doi:10.1371/journal.ppat.1002464

5. Delacollette C, Bustos MD, Thimasarn K, Ortega L, Christophel EM, Ringwald P. Antimalarial and artemisinin resistance in the Greater Mekong subregion. Regional Health Forum 2011;15:131-133. 